\title{
Modeling dynamic interactions in supply chains using agent- based simulations
}

\author{
Mariana Dorigatti, Omar Chiotti, Héctor E. Salomone \\ INGAR-CONICET, Avellaneda 3657, S3002GJC Santa Fe, Argentina \\ \{marianadorigatti, chiotti, salomone\}@santafe-conicet.gov.ar
}

\begin{abstract}
In this work, we present preliminary results of our research on the construction of an agent-based simulation framework suitable to support the analysis of complex supply chain interactions as the one required for the performance assessment in collaborative supply chains. In particular we focus in the modeling of dynamic interactions through agent-to-agent message communication avoiding predefined supply chain network structures. For defining the internal structure of agents we explore the application of the SCOR reference model to bring a business process perspective and adopt the requirement of making explicit separation of the execution and control and decision making processes.
\end{abstract}

Keywords: agent-based simulation, collaborative supply chains, SCOR model

\section{$1 \quad$ Introduction}

A collaborative supply chain implies that two or more independent firms work jointly to plan and execute supply chain operations with increased performance than acting in isolation. The e-business environment enabled a series of collaboration mechanisms for information sharing, operation coordination and joint decision making that convey the promise of improving the competitive advantages for all the partners engaged in a common supply chain. (Swaminathan \& Tayur 2003)

Despite the strong theoretical arguments about the increased overall performance of these collaboration models, the actual adoption in practice is still rather limited to some known configurations where a dominant member of the supply chain set the pace for the collaboration extent.

One of the main hurdles for extending the collaborative models to other supply chains where members are more independent business partners, is the difficulty to perform a fair assessment of the distribution of benefits and efforts a given collaboration model will bring to the partnership.

While analytical models have provided very valuable insights, both qualitative and quantitative, to better understand the collaboration mechanisms, only simulation based approaches can afford the complexity of real scenarios.(H. Chan \& F. Chan 2010)

Building ad-hoc simulation models for studying complex supply chain interactions can be prohibitive both in terms of cost and time. Therefore availability of a simula- 
tion framework, easy to use by business managers, that facilitates the development of those models has a strong incentive in the quest of nowadays business efforts to increase their supply chain performance.

The objective of this work is to present some preliminary research progress in the construction of a systematic and re-usable agent-based simulation framework for supporting the analysis of collaborative interactions in supply chains.

The remainder of this work is structured as follows: Section 2 presents the related literature. A description of the framework is presented in Section 3. Section 4 presents a case study used for validation purpose. Section 5 describes the results of the case study, and, finally, conclusions are presented in Section 6.

\section{$2 \quad$ Related Literature}

Discrete Event simulations and Systems Dynamics have been the modeling approaches most widely used to analyze the behavior of supply chains. An extensive literature review, (Tako \& Robinson 2012) records the usage of both approaches in different problems of supply chain. Despite the large list of contributions, the study of dynamic supply relationships among independent members appears as barely explored. (Umeda \& Lee 2004) propose a generic hybrid-modeling framework that combines discrete-event simulations with system-dynamics simulations. Discrete event models represent operational processes within the supply chain; and system dynamic models represent reactions in supply chain management circumstances. Traditional discrete-event approaches usually adopt a network perspective and are focused on representing the supply chain's topology and infrastructure, while generally assuming implicit representations of the control and decision processes.

Agent based simulation offers a promising framework to capture the dynamics aspects of logistics coordination among supply chain partners. Agent based approaches focus on individual participant's behavior and decision processes, often at the expense of event-oriented aspects of supply chains, as well as more global activities and policies. A review of agent based formalisms for supply chain simulation can be found in (Chatfield et al. 2007). In this area, the work of (Swaminathan et al. 1998) outstands as one of the most comprehensive attempts to build a generalized framework. They were pioneers in having a vision of agent for the purpose of a flexible and reusable modeling and simulation framework that allows for the development of models to address issues related to configuration, coordination, and contracts. Models are made of reusable components representing different entities in the supply chain. Interaction protocols are introduced to support the agent's interactions by regulating the flow of materials, information and cash through message passing. Although in their library they classify the components as either structural or control elements, the proposed controlling structures fall short in providing explicit representations of the relationship between decision making activities and their corresponding execution actions.

Requirements for a simulation modeling framework suitable for supporting the analysis of collaborative supply chains have been thoughtfully described by (Van der 
Zee \& Van der Vorst 2005). In this work authors recognize the need for an explicit definition of control policies and coordination mechanisms. They also highlight the need for explicit definition of the timing and execution of decision activities. Upon these requirements they propose a modeling framework based in the key concepts of agents and jobs. The concepts of planning and control are explicitly represented through decision making agents carrying out control jobs. Although relationships between agents are governed by a generalized concept of flow (including materials, information and jobs), these interactions are less structured and far from well defined interaction protocols as it is desirable to have in a generalized and systematic agent based framework. Another limitation of this framework is that the internal structure of the agents is only defined at a very high level (jobs and resources) and the definition of actual supply chain entities are left to the specialization of this structure. The result is a rapid degradation of the generality conducing to premature ad-hoc components.

We note here that the adoption of a generalized and structured representation of the supply chain business processes is another important requirement for a reusable framework.

The Supply Chain Council has been developing and maintaining a process reference model, SCOR that has been widely adopted in the industry and proved the applicability of their standard processes to a broad range of different supply chains (Council 2012). The SCOR model appears as a natural reference for guiding the representation of the supply chain processes in systematic, generalized and business process oriented simulation framework. SCOR has been used before to guide the development of simulation models for analyzing supply chains. (Buckley \& An 2002) describe the use of SCOR concepts in the IBM's Supply Chain Analyzer tool. (Barnett \& Miller 2000) describe the architectural components used to implement a distributed supply chain modeling tool (e-SCOR) and its applications to demonstrate how enterprises are modeled and analyzed to determine the validity of alternative virtual business models. Using SCOR concepts, (Pundoor \& Herrmann 2004) build simulation models that integrate discrete event simulation and spreadsheets. Simulation models are hierarchical and include sub-models that capture activities specific to supply chains. In this framework, a supply chain simulation model has three levels. The first level is the simulation model. The second level has sub-models that correspond to supply chain participants (consumers, producers, and traders). The third level has submodels that correspond to process elements (across all process categories) that each participant performs. Each process element is implemented as a separate sub-model that represents a specific activity in a supply chain. For building the supply chain simulation model, these modules are gathered together and connected using standard interfaces that represent material, information, and cash flow.

Another important requirement for a simulation framework aimed at supporting the dynamics of business interaction among supply chain partners is the representation of structured collaborative business processes. This aspect has not been developed by the literature in supply chain simulation. In this regards the work of (Stuit \& Szirbik 2009) in formalizing the interaction and behavior of agents engaged in collaborative business processes provide useful concepts for this representation. 


\section{Framework description}

In this section we describe the simulation framework that we are creating to address the requirements discussed to support the analysis of collaborative interactions in supply chains by means of agent based simulations. These requirements can be summarized as follows:

i) Provide re-usable components that can be easily assembled to set a wide variety of supply chain scenarios among independent partners.

ii) Allow for establishing dynamic links among partners without requiring predefined network structure.

iii) Adopt a business process oriented perspective to reflect the activities in the supply chain following the SCOR reference model

iv) Provide for explicit representations of control and decision making activities separated from the execution activities.

v) Support the interaction among agents by using message based and document oriented protocols that resemble actual business interactions.

vi) Allow the deployment of diverse implementations of the internal processes of each member with independence of the others by using standard contractual interfaces.

vii) Include the assessment of standard performance indicators.

To fulfill these requirements a set of goals was defined. Based on these goals, subgoals, main actions and data needed for achieving them were identified (Table 1). Prometheus was used for guiding the development process (Sterling, S \& Taveter 2009).

\begin{tabular}{|c|c|c|c|}
\hline Goal & Sub-goal & Actions & Involved data \\
\hline \multirow{2}{*}{$\begin{array}{r}\text { Plan } \\
\text { Source }\end{array}$} & \multirow{2}{*}{$\begin{array}{c}\text { Establish } \\
\text { sourcing plan }\end{array}$} & \multirow{2}{*}{$\begin{array}{l}\text { Decide the replenishments } \\
\text { orders placed to every provider }\end{array}$} & List of source materials \\
\hline & & & List of source orders \\
\hline \multirow{2}{*}{$\begin{array}{l}\text { Plan } \\
\text { Make }\end{array}$} & \multirow{2}{*}{$\begin{array}{l}\text { Establish Pro- } \\
\text { duction plan }\end{array}$} & \multirow{2}{*}{$\begin{array}{l}\text { Deciding the production or- } \\
\text { ders issued for manufacturing }\end{array}$} & List Production Orders \\
\hline & & & List of BOM \\
\hline \multirow{2}{*}{$\begin{array}{l}\text { Execution } \\
\text { Source }\end{array}$} & \multirow{2}{*}{$\begin{array}{l}\text { Receive Prod- } \\
\text { uct }\end{array}$} & \multirow{2}{*}{$\begin{array}{l}\text { Receives the item and add it } \\
\text { to the list of source materials }\end{array}$} & List of source materials \\
\hline & & & List of source orders \\
\hline \multirow{2}{*}{$\begin{array}{l}\text { Execution } \\
\text { Make }\end{array}$} & \multirow[b]{2}{*}{ Produce } & \multirow{2}{*}{$\begin{array}{l}\text { Responsible for executing the } \\
\text { production orders. }\end{array}$} & List of make BOMs \\
\hline & & & $\begin{array}{l}\text { list of make Production } \\
\text { Orders }\end{array}$ \\
\hline \multirow{4}{*}{$\begin{array}{l}\text { Execution } \\
\text { Deliver }\end{array}$} & \multirow{2}{*}{$\begin{array}{l}\text { Receive Cus- } \\
\text { tomer Orders }\end{array}$} & \multirow{2}{*}{$\begin{array}{l}\text { Accept orders from its con- } \\
\text { sumer }\end{array}$} & list of deliver materials \\
\hline & & & list of order places \\
\hline & \multirow{2}{*}{ Ship Products } & \multirow{2}{*}{ Send items requested } & list of deliver materials \\
\hline & & & list of order places \\
\hline
\end{tabular}

Table 1: Goals, sub-goal, actions and data

Following, agents responsible for meeting these objectives were defined. Figure 1 describes sub-goals and agents responsible for achieving them. Sub-goals can be achieved by the participation of an agent (e.g. Establish Sourcing Plan) or by the par- 
ticipation of two agents working in a coordinated way (e.g. Establish Production Plan). The framework is composed of three types of agent: SCMember, SCSource, SCCustome.

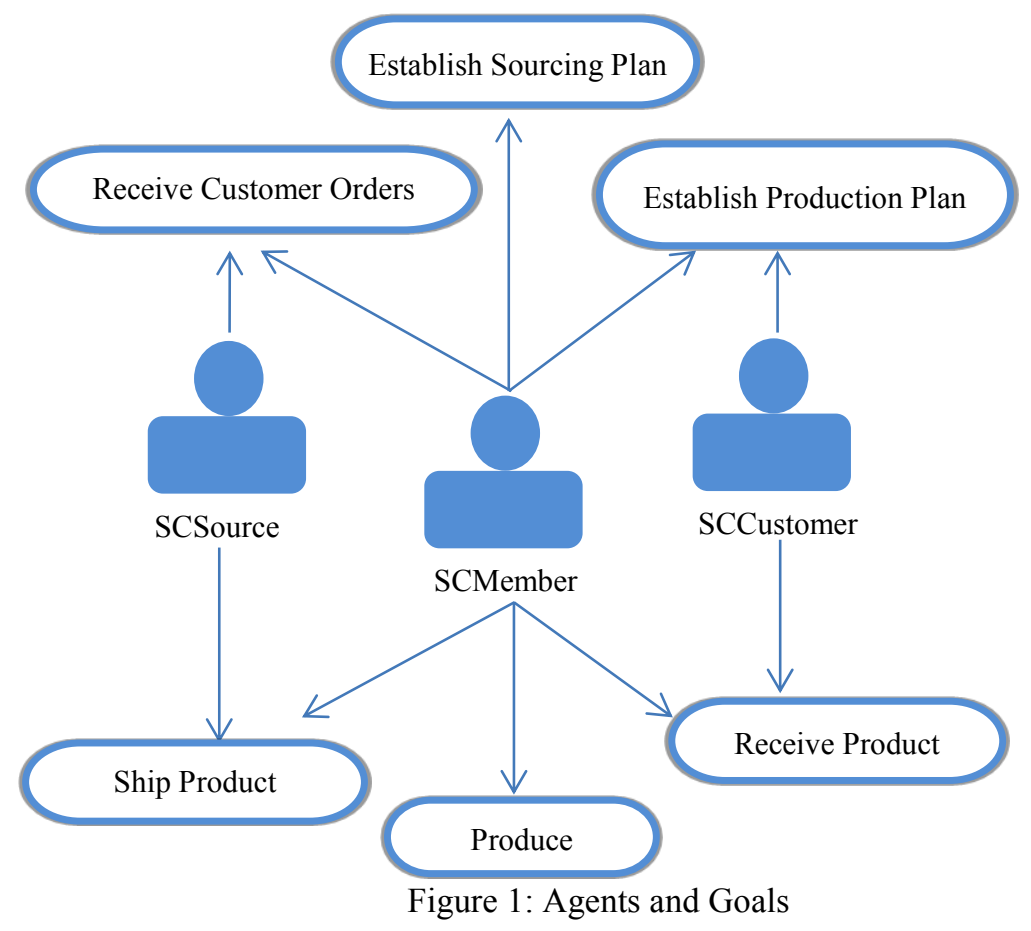

Every supply chain partner in the framework is represented by an agent SCMember deploying a collection of supply chain business processes in the sense of an SCOR organization. Each SCMember is responsible for providing the Source, Make and Deliver processes both at the level of Plan and Execution.

The SCMember is also responsible for exposing the interfaces that supports business interaction in the form of service oriented ports. At this early stage of the framework evolution we have included the "input" service port, to account for the reception of materials and the "order" service port to account for the exchange of documentbased messages.

The agent SCMember manages two lists of SKU objects. One for representing products that are shipped and the other materials used to produce its final products. The SKU object represents a stocking keeping unit and maintains the variables for accounting the item inventory, the backlog, a list of providers for its replenishment and parameters for the inventory control policy: minimum inventory, replenishment lot size, and demand modeling parameters. The SKU object is also responsible for keeping a list of possible providers and their performance. This performance is used to dynamically decide to which SCMember the replenishment orders will be placed. Figure 2 illustrates the modeling elements included in the SKU object. 
The agent SCMember implemented so far includes the business process to represent a make-to-stock organization that handles a list of source materials (the list source_SKUs) and a list of orders issued to the provider to replenish these materials (the list source orders). It also offers an implementation for the SCOR business processes: sP2.4.EstablishingSourcingPlan at the Plan Source level which is the process to decide the replenishment orders placed to every provider and $s$ S1.2.ReceiveProduct at the Execution Source level.

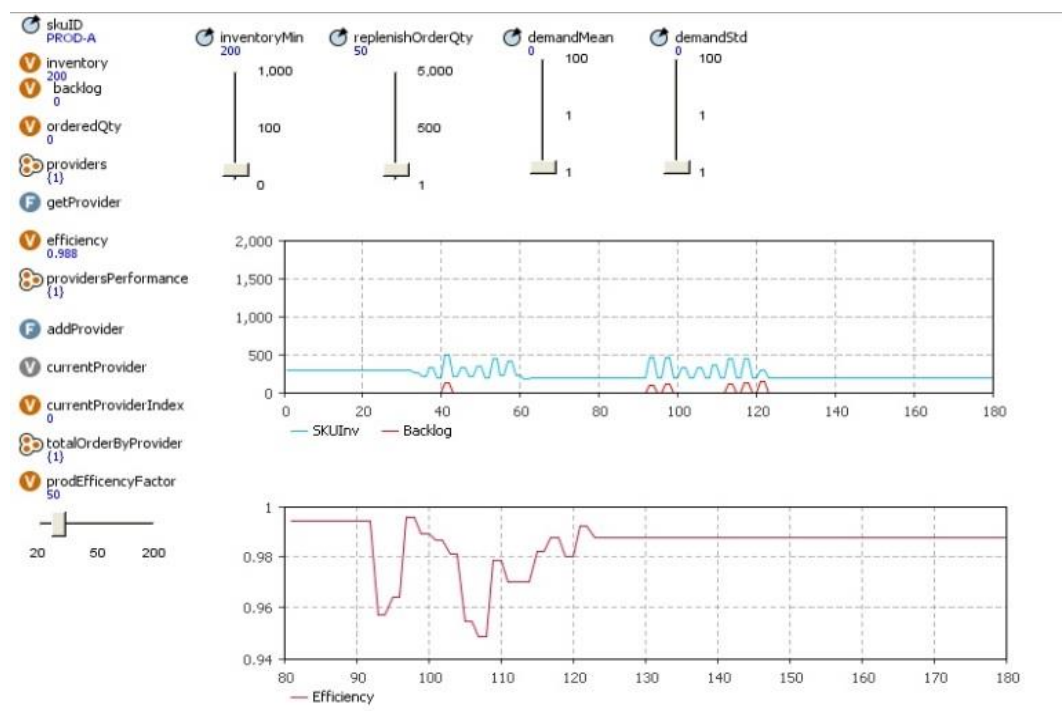

Figure 2: SKU object

For the Make function, the agent maintains a list of production orders and a list of BOMs (Bill-Of-Materials) that define the relationship among the units of materials consumed for producing a unit of products. It offers implementations for the SCOR business processes sP3.4.EstablishingProductionPlan at the Plan Make level that is responsible for deciding the production orders issued for manufacturing. At the Plan execution level, it implements the process $s M 1.3$ Produce, which is responsible for executing the production orders.

For the Deliver function, the agent in the current implementation behaves as a demand reactive partner, able to accept orders from its consumer and ship them as soon the ordered items are available. It handles a list of delivery materials (the list deliver_SKUs) and a list of orders places by its consumers (the list delivery_orders) It implements the SCOR processes sD1.2.ReceiveCustomerOrders and sD1.12.ShipProducts. The modeling elements of the SCMember agent are illustrated in table 1.

The framework was implemented using AnyLogic (XJ Technologies 2012). This is a multi-paradigm simulation modeling environment that supports hybrid combina- 
tions of System Dynamics, Process-centric (discrete event), and Agent Based modeling. Figure 3 depicts the configuration of the internal structure of agent SCMember.

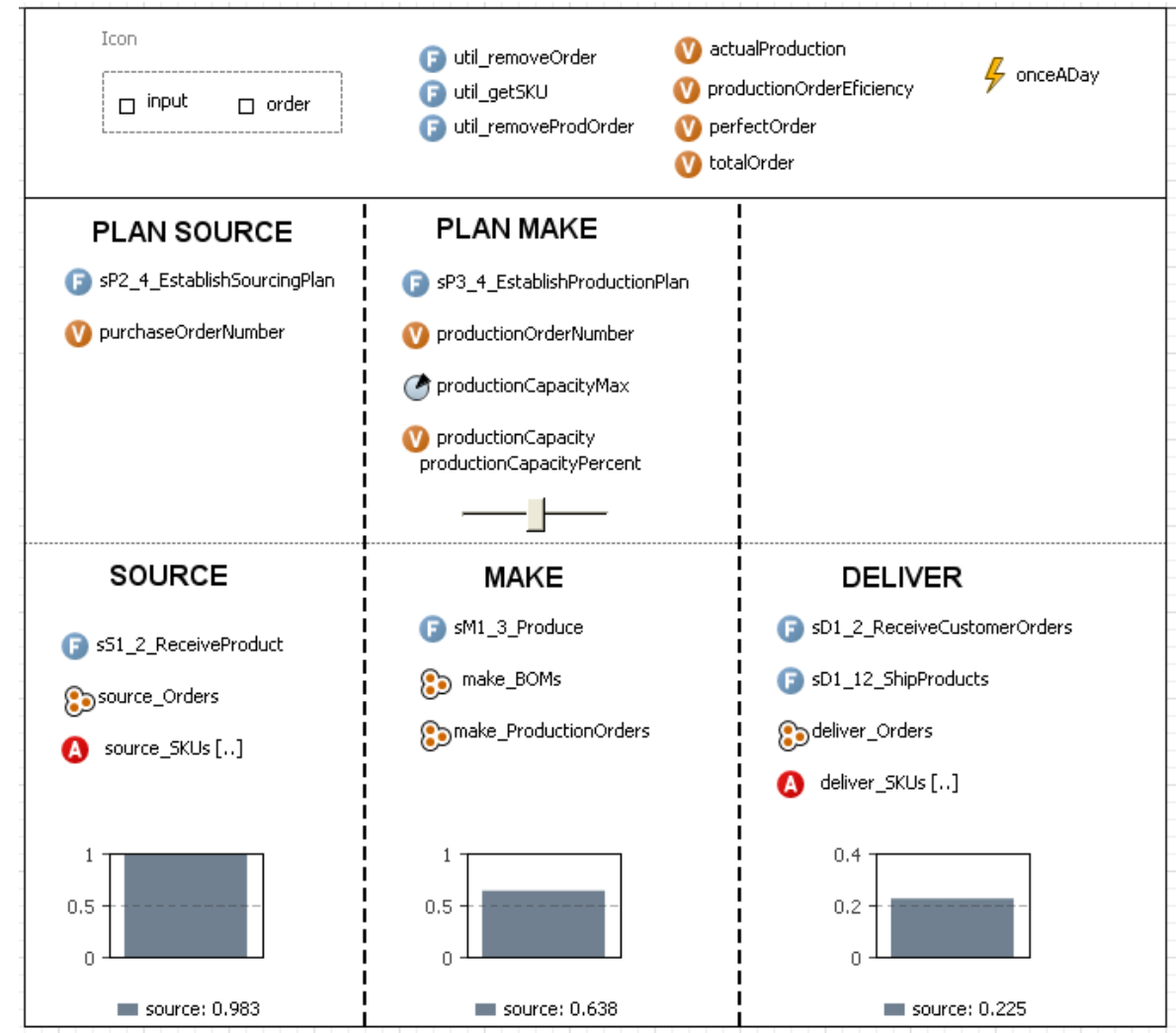

Figure 3: internal structure of agent SCMember

The interaction among SCMember agents is achieved by delivering messages to the corresponding service port. Messages have attached a business document including all information needed to its interpretation. For instance, the message for placing a replenishment order includes the following fields:

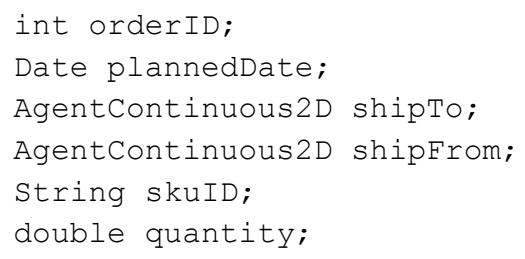

The reception of products is also communicated through a message containing the order that is being delivered. 
The timing of the actions is triggered either by scheduled events: for instance, the process sP3.4.EstablishingProductionPlan is scheduled to be performed once a day by using a cyclic event. Other actions are triggered upon the arrival of a certain message in a service port. For instance, the process sS1.2.ReceiveProduct is performed each time a message is delivered in the input service port.

For representing boundary entities of the supply chain, we included two additional agents: SCSource and SCCustomer. They can be interpreted as specialized SCMembers implementing only a subset of business processes. SCSource does only implement the Delivery processes including the order service port and the SCCustomer does only implement the Source processes including the input service port. Additionally, the SCCustomer agent includes functions to keep a record of its provider's performance and uses this record to select the current provider for every source SKU (Figure 4).

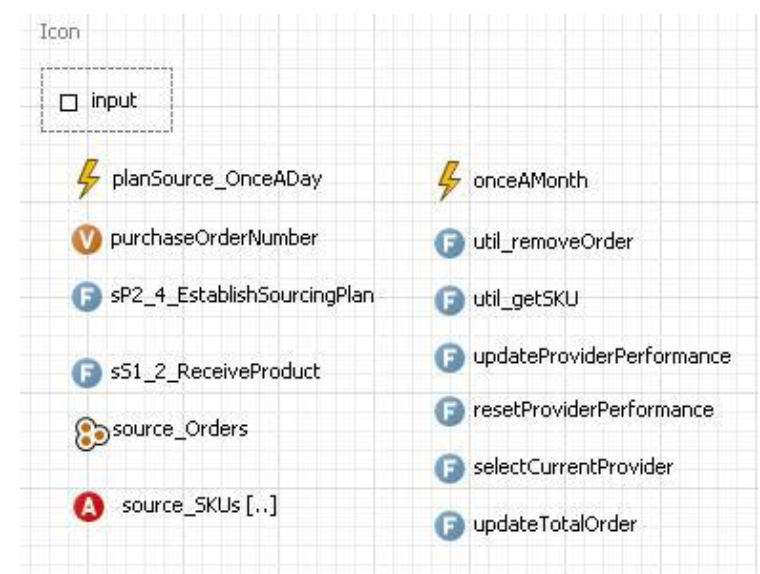

Figure 4: SCCustomer agent

\section{$4 \quad$ Validation using a Case Study}

A case study (Figure 5) was built to perform a preliminary validation of the proposed framework. The case represents a supply chain consisting of four organizations producing and consuming the materials indicated in Table 2:

\begin{tabular}{|l|l|l|}
\hline Member & Produces and Deliver & Consumes \\
\hline Producer1 & PROD & MAT-1, MAT-2 \\
\hline Producer2 & PROD & MAT-1, MAT-2 \\
\hline Supplier1 & MAT-1 & RM \\
\hline Supplier2 & MAT-2 & RM \\
\hline \multicolumn{2}{|c|}{ Table 2: material consumed and produced }
\end{tabular}


Producer1 and Producer 2 are essentially competitors offering the same products to the consumer. Supplier1 and Supplier2 are providers of materials required for both producers. These four organizations are modeled by a SCMember agent.

There is a single SCSource agent named Source providing raw material RM and it is considered as a boundary provider not included in the supply chain. An agent of type SCCustomer named Customer represents the marketplace.

Customer consumes the product $P R O D$ by placing orders to a selected producer. Customer demand is modeled as a non-deterministic quantity following a normal distribution.

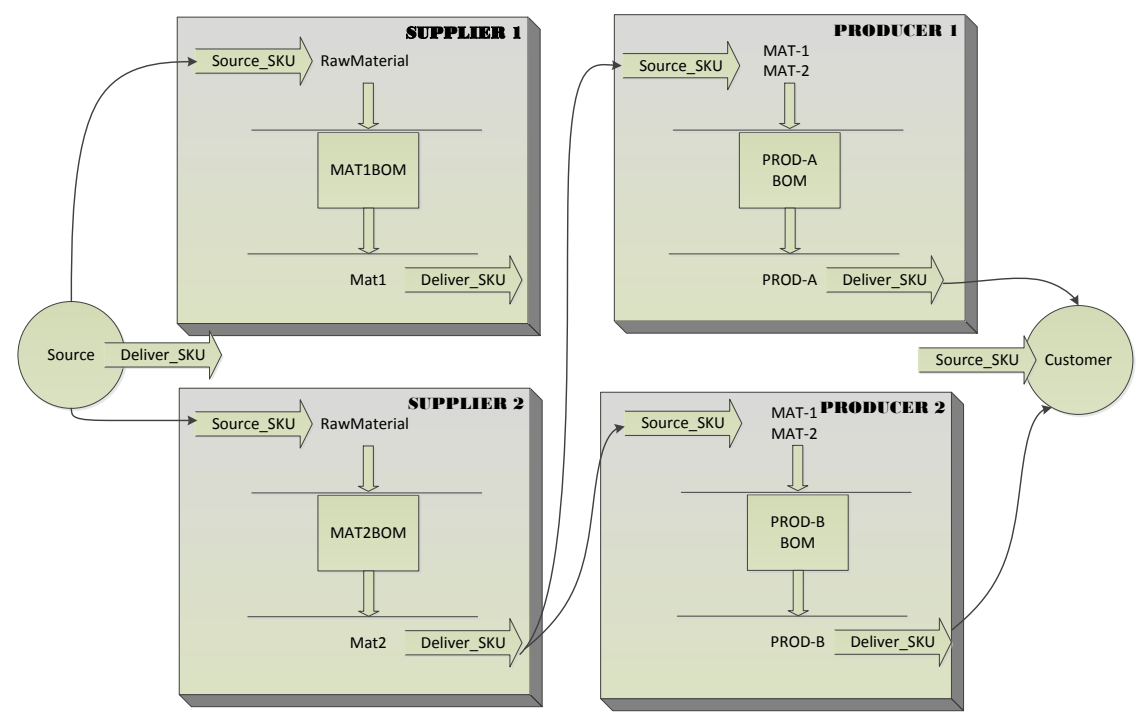

Figure 5: Case Study

The selected producer is chosen from a list through the function selectCurrentProvider. This choice is updated every month by a scheduled event. This function allows selecting each month the provider with the best delivery performance. Producerl is set to have a better performance under normal operation conditions, so it will be the preferred one unless is experiencing a disruption. Every month, both producers reset to have a $100 \%$ performance and this index is discounted every time an order is shipped with delay. At the end of each month, the selection is revised and if the alternative provider improves the current one by a $5 \%$ index, then selection is changed.

The case is simulated over a period of 180 days and it has one disruptive event forcing Producer 1 to reduce its production capacity at 50\% during 65 days. This disruption is scheduled to occur at day 60 .

The intention of setting this scenario is to force the performance index of Producerl to deteriorate and illustrate the switch to the alternative provider. 


\section{$5 \quad$ Results}

Based on the orders pulled by Customer following its demand normal distribution, every member in the supply chain applied its independent replenishment plan. Table 2 summarizes the orders exchanged after 180 days of operations.

\begin{tabular}{|c|c|c|}
\hline Members & Arrived Supply Orders & Dispatched Customer Orders \\
\hline Supplier 1 & 36 & 41 \\
\hline Supplier 2 & 59 & 80 \\
\hline Producer 1 & 110 & 153 \\
\hline Producer 2 & 11 & 30 \\
\hline
\end{tabular}

Table 3: Result of amount orders

During the first 60 days, Producer 1 keeps the preferred provider position (Figure 6). Although its performance index is decreasing along every month, the potential improvement of Producer 2 is not enough to force the swap. After the disruptive event forcing the reduction in the production capacity of Producerl, its performance index worsens very quickly and at the end of month 3 , Producer 2 is selected as the preferred provider instead.

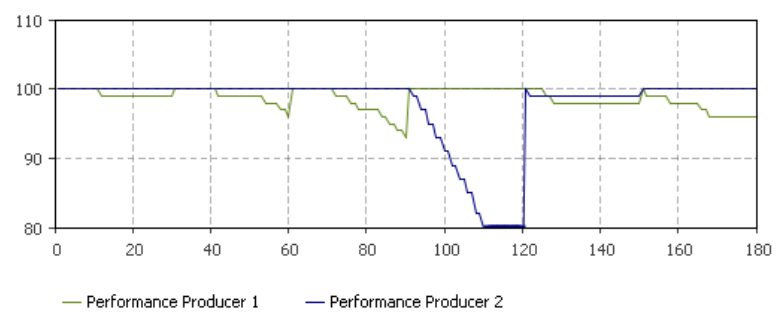

Figure 6: Performance index of the Producers

After Producer1 resumes its full production capacity, it becomes again the preferred provider as it has a better performance.

In Figures 7 and 8, the inventory and backlog variation is shown for both producers:

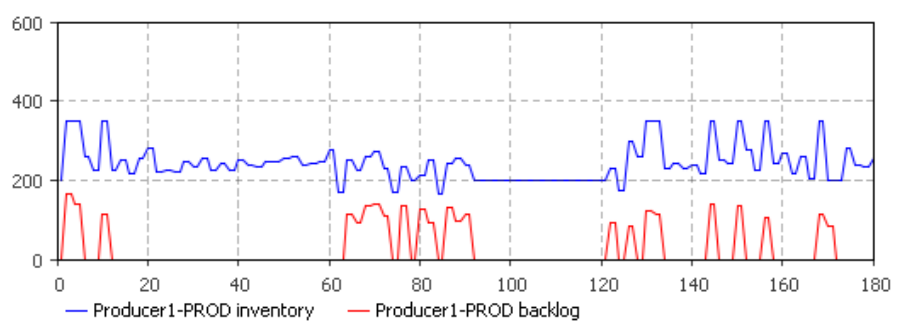

Figure 7: Inventory and backlog of Producer 1 


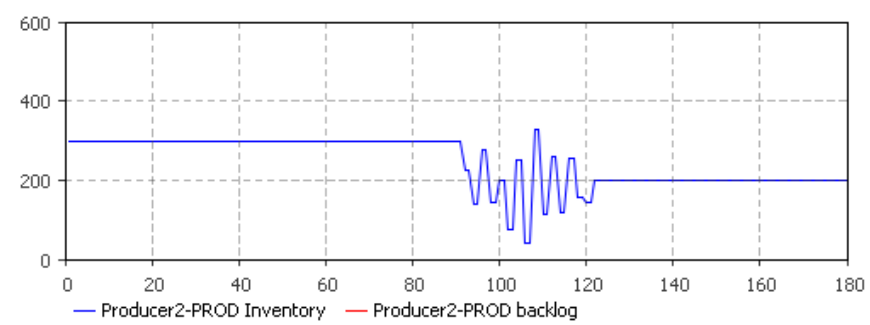

Figure 8: Inventory and backlog of Producer 2

The effects of these changes in the Customer preference can be analyzed as they propagate upstream in the supply chain. For instance, in the inventory variation of Material MAT2 in both producers (Figure 9):
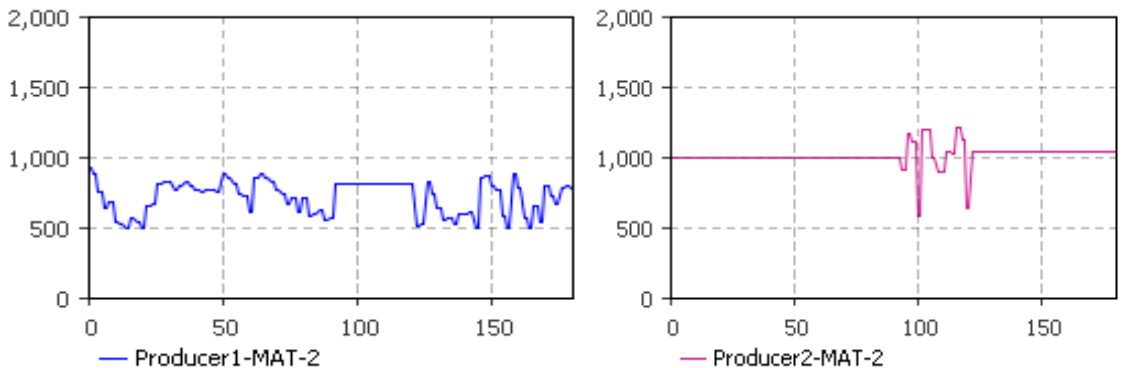

Figure 9: Inventory of MAT-2 at both producers

And finally, how that variations affect the Supplier2 (Figure 10):

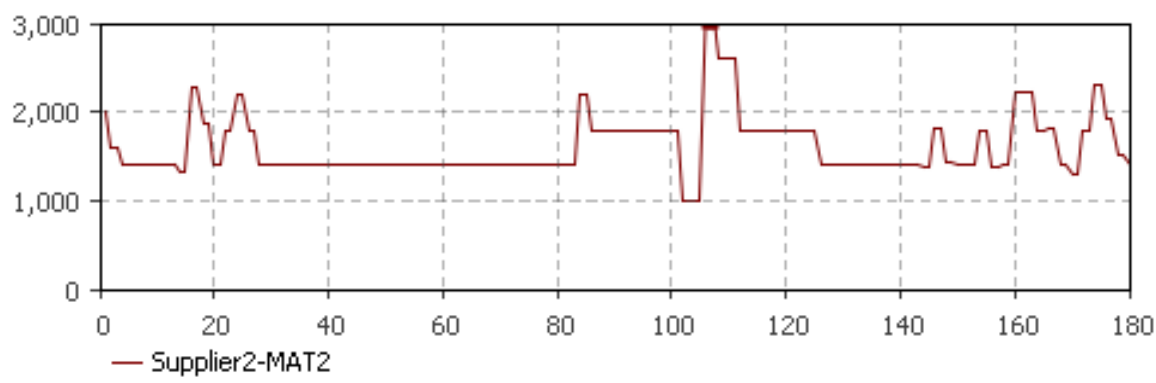

Figure 10: Inventory of MAT-2 at Supplier2

\section{Conclusions}

We have presented an agent based simulation framework designed to fulfill the requirements for analyzing complex relationships in multi-organizational supply chains. 
The proposed design builds on top the previous proposals that have adopted an explicit separation of the processes for execution, control and decision making and proposes re-usable component architecture. A business process oriented perspective is adopted to organize the internal behavior of the agents though the implementation of SCOR standard process.

The main focus of the presented case study is to illustrate the framework capability to handle dynamic links among supply chain partners. It should be noted that the definition of the supply network does not requires providing fixed connections among the interacting entities.

Message oriented interactions through services ports exposed by the agents enable the establishment of the customer-provider relationship in run-time. In the case study this feature is demonstrated by the ability of Customer to swap the preferred provider upon reaction to a give service level.

Components reusability is demonstrated by creating a four members supply chain resorting to the same basic agent initialized with its own independent parameters. Every member is independent to generate its own Plan, Source, Make and Deliver processes, separating the execution aspects from those related to decision and control.

These capabilities are regarded as crucial to provide the analytical support needed to study and assess realistic collaborative models in supply chains.

\section{$7 \quad$ References}

Barnett, M.W. \& Miller, C.J., 2000. ANALYSIS OF THE VIRTUAL ENTERPRISE USING DISTRIBUTED SUPPLY CHAIN. , pp.352-355.

Buckley, S. \& An, C., 2002. 2 Supply Chain Simulation.

Chan, H. \& Chan, F., 2010. A REVIEW OF COORDINATION STUDIES IN THE CONTEXT OF SUPPLY CHAIN DYNAMICS. International Journal of Production Research, 48(10), pp.2793-2819.

Chatfield, D.C., Hayya, J.C. \& Harrison, T.P., 2007. A multi-formalism architecture for agent-based, order-centric supply chain simulation. Simulation Modelling Practice and Theory, 15(2), pp.153-174. Available at: http://linkinghub.elsevier.com/retrieve/pii/S1569190X06000761 [Accessed January 28, 2013].

Council, S., 2012. Supply chain operations reference model-Revision 11.0, Available at: http://www.leanportal.sk/Files/Modely/SCOR.pdf [Accessed February 21, 2013].

Pundoor, G. \& Herrmann, J.W., 2004. A hierarchical approach to supply chain simulation modeling using the Supply Chain Operations Reference model. 
Sterling, S, L. \& Taveter, K., 2009. The art of agent-oriented modeling, The MIT Press. Available at: http://mitpress.mit.edu.

Stuit, M. \& Szirbik, N.B., 2009. Towards agent-based modeling and verification of collaborative business processes: an approach centered on interactions and behaviors. International journal of cooperative information ..., 18(03n04), pp.423-479. Available at:

http://www.worldscientific.com/doi/abs/10.1142/S0218843009002063 [Accessed April 12, 2013].

Swaminathan, J.M., Smith, S.F. \& Sadeh, N.M., 1998. Modeling Supply Chain Dynamics : , 29(3), pp.607-632.

Swaminathan, J.M. \& Tayur, S.R., 2003. Models for supply chains in e-business. Management Science, 49(10), pp.1387-1406.

Tako, A. a. \& Robinson, S., 2012. The application of discrete event simulation and system dynamics in the logistics and supply chain context. Decision Support Systems, 52(4), pp.802-815. Available at: http://linkinghub.elsevier.com/retrieve/pii/S0167923611002211 [Accessed February 28, 2013].

Umeda, S. \& Lee, Y., 2004. Design specifications of a generic supply chain simulator. ... of the 36th conference on Winter simulation. Available at: http://dl.acm.org/citation.cfm?id=1161946 [Accessed January 28, 2013].

XJ Technologies, 2012. Anylogic 6.

Van der Zee, D.J. \& Van der Vorst, J.G. a. J., 2005. A Modeling Framework for Supply Chain Simulation: Opportunities for Improved Decision Making*. Decision Sciences, 36(1), pp.65-95. Available at: http://doi.wiley.com/10.1111/j.1540-5915.2005.00066.x. 